ARTICLE

\title{
Host-guest chemistry for tuning colloidal solubility, self-organization and photoconductivity of inorganic-capped nanocrystals
}

\author{
Maryna I. Bodnarchuk ${ }^{1,2}$, Sergii Yakunin ${ }^{1,2}$, Laura Piveteau ${ }^{1,2} \&$ Maksym V. Kovalenko ${ }^{1,2}$
}

Colloidal inorganic nanocrystals (NCs), functionalized with inorganic capping ligands, such as metal chalcogenide complexes (MCCs), have recently emerged as versatile optoelectronic materials. As-prepared, highly charged MCC-capped NCs are dispersible only in highly polar solvents, and lack the ability to form long-range ordered NC superlattices. Here we report a simple and general methodology, based on host-guest coordination of MCC-capped NCs with macrocyclic ethers (crown ethers and cryptands), enabling the solubilization of inorganiccapped NCs in solvents of any polarity and improving the ability to form NC superlattices. The corona of organic molecules can also serve as a convenient knob for the fine adjustment of charge transport and photoconductivity in films of NCs. In particular, high-infrared-photon detectivities of up to $3.3 \times 10^{11}$ Jones with a fast response ( $3 \mathrm{~dB}$ cut-off at $3 \mathrm{kHz}$ ) at the wavelength of $1,200 \mathrm{~nm}$ were obtained with films of PbS/ $\mathrm{K}_{3} \mathrm{AsS}_{4} /$ decyl-18-crown-6 NCs.

\footnotetext{
${ }^{1}$ Laboratory of Inorganic Chemistry, Department of Chemistry and Applied Biosciences, ETH Zürich, Vladimir Prelog Weg 1, CH-8093 Zürich, Switzerland.

${ }^{2}$ Laboratory for Thin Films and Photovoltaics, Empa-Swiss Federal Laboratories for Materials Science and Technology, Überlandstrasse 129,

CH-8600 Dübendorf, Switzerland. Correspondence and requests for materials should be addressed to M.I.B. (email: bodnarchuk@inorg.chem.ethz.ch) or to M.V.K. (email: mvkovalenko@ethz.ch).
} 
$\mathrm{N}$ ew strategies for the programmable, scalable and experimentally facile bottom-up design of solid-state materials from chemically synthesized nanocrystals (NCs) are becoming increasingly important because of their promising applications in electronic and optoelectronic devices ${ }^{1-6}$. In this regard, NC surface chemistry has become a major bottleneck and, at the same time, a major enabler due to its crucial influence on nearly all of the physical and chemical properties of $\mathrm{NCs}^{5,7}$. Bulky and electrically insulating organic capping ligands (surfactants) enable the formation of monodisperse colloidal NCs. In the direction towards subsequent applications of NCs in solid-state devices, the last several years have seen a surge of reports showing the great potential of small, electrically conductive inorganic capping ligands, such as metal chalcogenide complexes (MCCs, for example, $\mathrm{Sn}_{2} \mathrm{~S}_{6}{ }^{4-}, \mathrm{SnTe}_{4}{ }^{4-}, \mathrm{Cu}_{7} \mathrm{~S}_{4}^{-}, \mathrm{In}_{2} \mathrm{Se}_{4}{ }^{2-}$ and $\left.\mathrm{AsS}_{4}{ }^{3-}\right)^{8-20}$ and other ionic inorganic moieties (for example, halides, pseuodohalides, metal-free chalcogenido, hydroxyl and amido ions, oxo- and polyoxometallates, and halometalates) $)^{12,17,21-26}$. All of these ligands have been used to displace initial organic capping ligands, forming stable colloidal dispersions. Another powerful strategy, applicable to metal oxide and chalcogenide NCs, is the formation of cationic and naked, but colloidally stable NCs upon controlled stripping of the strongly bound native organic ligands with the simultaneous coordination of metal cations on the NC surface with weakly nucleophilic ligands ${ }^{26-28}$. Such all-inorganic NC surface chemistries have enabled major advances in the design of solidstate NC devices: high electronic mobilities in densely packed NC films $\left(5-35 \mathrm{~cm}^{2} \mathrm{~V}^{-1} \mathrm{~s}^{-1}\right)^{12,16,22,29-33}$, the integration of highly luminescent $\mathrm{PbS} / \mathrm{CdS} \mathrm{NCs}$ into fully inorganic infrared transparent matrices ${ }^{13}$, efficient photoconductivity in the visible and near-infrared regions with detectivities reaching $10^{13}$ Jones $^{34}$, improved electrocatalytic properties of CdSe NCs (hydrogen evolution ${ }^{26}$ and enhanced electronic transport in NC-based Li-ion batteries ${ }^{25}$. Inorganic-capped NCs also served as fully inorganic inks and precursors for $\mathrm{CuInS}_{2}, \mathrm{Cu}\left(\mathrm{In}_{1-x} \mathrm{Ga}_{x}\right) \mathrm{Se}_{2}$, $\mathrm{Cu}_{2} \mathrm{ZnSn}(\mathrm{S}, \mathrm{Se})_{4}$ and $\mathrm{PbS}$ phases as thin-film absorbers for photovoltaics ${ }^{11,35,36}$, CdSe thin films with exceptionally high electron mobilities of up to $200 \mathrm{~cm}^{2} \mathrm{~V}^{-1} \mathrm{~s}^{-1}$ (ref. 37), ionically conductive composites of $\mathrm{Ag}_{2} \mathrm{~S}$ NCs embedded into a $\mathrm{GeS}_{2}$ matrix ${ }^{38}, \mathrm{Sb}_{2} \mathrm{Te}_{3}$-based nanocomposites for thermoelectrics ${ }^{20,39}$, and NC-in-glass windows with electrochemically tunable transmittance ${ }^{40,41}$. MCCs have been employed as capping ligands in the majority of these reported examples of solid-state devices/materials. The versatility of MCCs as ligands is rooted in their exceptionally strong binding affinity to NC surfaces, including commonly studied NCs of metals ( $\mathrm{Au}$ and $\mathrm{Pd}$ ) and semiconductors (CdSe, PbS, InAs, InP and so on). Ligand exchange with MCCs proceeds quantitatively to completion in most cases and leads to the phase transfer of NCs from nonpolar to highly polar solvents, such as hydrazine or $\mathrm{N}$-methylformamide (MFA), forming concentrated, long-term stable colloidal solutions. In such highly polar media $(\varepsilon=40-190)$, the strong adsorption of anions combined with the dissociation of cations leads to efficient electrostatic colloidal stabilization. Straightforward solution-based processing with common techniques such as spin- or spray-coating has led to the aforementioned success of inorganic-capped NCs in solidstate devices. At the same time, MCC-capped NCs lack the ability to disperse in moderately polar and apolar solvents and/or to mix with molecules of various polarities, and exhibit limited ability to form regular and ordered NC solids.

Herein we demonstrate a simple approach to continuously tune the surface functionality of MCC-capped NCs from highly polar to intermediately and finally rather nonpolar.
Consequently, the range of solvents and other media in which inorganic-capped NCs can be dispersed is greatly extended, and includes, for instance, common volatile organic solvents such acetonitrile, acetone and toluene. This is achieved here by the host-guest coordination of MCC-capped NCs with macrocyclic ethers-crown ethers ${ }^{42}$ and cryptands ${ }^{43}$-as host molecules for the alkali metal counter ions of MCCs, as depicted in Fig. 1. This approach turns the cationic side of the all-inorganic surface capping into an important factor for adjusting chemical and physical properties of NCs in the colloidal state and in thin films. Since the macrocycle molecule is neutral, and the stability of the resulting complex is highly solvent dependent, the macrocycle can be removed from the NC film by merely dipping it into the 'wrong' solvent. Instead of being removed, this organic corona can also be used for fine-tuning of the charge transport, as demonstrated here for the photoconductive properties of $\mathrm{PbS}$ NCs. In films of $\mathrm{PbS} / \mathrm{K}_{3} \mathrm{AsS}_{4} /$ decyl-18-crown-6 NCs, high-infrared-photon detectivities of up to $3.3 \times 10^{11}$ Jones with a fast response $(3 \mathrm{~dB}$ cut-off at $3 \mathrm{kHz})$ at the wavelength of $1,200 \mathrm{~nm}$ were obtained. On the other hand, films of crown-free $\mathrm{PbS} / \mathrm{K}_{3} \mathrm{AsS}_{4} \mathrm{NCs}$ are excessively conductive and therefore not suitable for observing efficient photocurrent generation, while the initial oleate-capped $\mathrm{PbS}$ NCs are fully insulating, precluding photocurrent measurements.

\section{Results}

Host-guest approach to tune the solubility of NCs. Multiple etheral oxygen atoms are capable of coordinating metal cations. Owing to such dipole-ion interactions and geometric effects, cyclic (crown ethers) and polycyclic (cryptands) polyether molecules are known to selectively coordinate very small and hard alkali metal cations $\left(\mathrm{Li}^{+}, \mathrm{Na}^{+}\right.$and $\mathrm{K}^{+}$), enclosing them within the interior of donut-shaped rings (crowns) or cages (cryptands). Since the pioneering work in the 1960s on crown ethers by Pedersen ${ }^{42}$ and on cryptands by Lehn ${ }^{43}$, these host molecules have been used in organic and inorganic chemistry as phase transfer and solubilizing agents (especially for isolating peculiar compounds such as electrides, alkalides and Zintl phases), as phase transfer catalysts, or as discerning host molecules in ion-selective electrodes ${ }^{44}$. 'Host-guest' coordination has launched modern supramolecular chemistry and still stirs the imaginations of many scientists today, towards

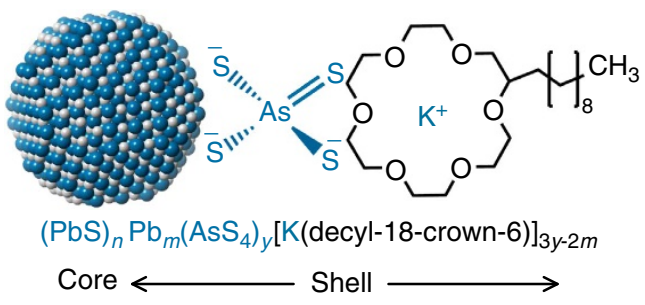

Other macrocycles:

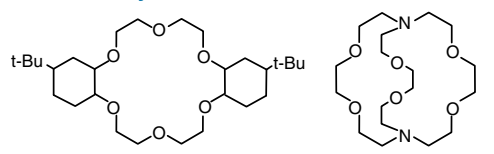

Figure 1 | Schematic of the complexation of inorganic-capped colloidal NCs with crown ethers or cryptands. The binding of a neutral macrocyclic polyether, decyl-18-crown-6, onto the surface of a PbS NC capped with $\mathrm{K}_{3} \mathrm{AsS}_{4}$ occurs via dipole-ion interactions. The entire assembly is held together by both the strong ion pairing and moderately strong host-guest interactions. The corona of macrocyclic molecules governs the dispersibility of such NCs in various solvents. 
boundless molecular structures and applications ${ }^{45,46}$. Just as in Pedersen's classic experiment demonstrating the utility of crown ethers for the solubilization of $\mathrm{KMnO}_{4}$ in benzene, leading to socalled 'purple' benzene due to the formation of lipophilic $[\mathrm{K}(18$ crown-6)] $\mathrm{MnO}_{4}$, we demonstrate in this work a practical route to the solubilization of MCC-capped colloidal NCs in moderately polar or fully nonpolar solvents using similar approach. For one selected system, $\mathrm{PbS} \mathrm{NCs} / \mathrm{K}_{3} \mathrm{AsS}_{4}$ ligand/decyl-18-crown-6 macrocycle (Fig. 1), we present a detailed characterization and discuss the key aspects of this supramolecular approach and its effect on solubility in various solvents, self-assembly into longrange ordered superlattices and (photo)conductive properties. We also briefly discuss other NC-ligand-macrocycle combinations tested in the course of this work.

The initial carboxylate-capped 3.5-10 nm PbS NCs consist of a stoichiometric $\mathrm{PbS}$ core covered with a $\mathrm{Pb}$-oleate monolayer ${ }^{47}$. After ligand exchange, elemental analysis of $4 \mathrm{~nm} \mathrm{PbS}$ NCs using Rutherford backscattering spectrometry (RBS) indicates that the $\mathrm{Pb}$-rich stoichiometry is retained, confirming that oleate anions are stripped off the surface and replaced with polydentate $\mathrm{AsS}_{4}{ }^{3}$ - ions. The remaining charge balance is provided by $\mathrm{K}^{+}$ cations. Such NCs are only dispersible in highly polar solvents (for example, MFA with a static dielectric constant that is a factor of 2 higher than that of water) since efficient electrostatic dissociation of $\mathrm{K}^{+}$counter ions is required. Here we use these $\mathrm{K}^{+}$cations to attach charge-neutral macrocycles via weak host- (macrocycle) guest $\left(\mathrm{K}^{+}\right)$interactions, as depicted in Fig. 1. As a result, the surface chemistry of the NCs becomes rather amphiphilic, that is, making them dispersible in both polar and nonpolar media, due to electrostatic and steric effects. The relative contributions of electrostatics and steric interactions between the NCs and with the solvent are dependent on the selection of macrocycle and solvent, as discussed below. In a typical procedure, inorganic-capped NCs are precipitated from MFA by adding acetonitrile or an acetonitrile/toluene mixture, separated by centrifuging, and redispersed in acetonitrile containing decyl-18-crown-6 (decyl-18C6, 0.1 M), instantly forming a colloidally stable solution. Acetonitrile is chosen as a solvent since it exhibits high stability constants of host-guest complexes, reported to be among the highest for all tested macrocycles. It is important to note that inorganic-capped NCs cannot be dispersed in acetonitrile in the absence of macrocycles. To disperse 'crowned' NCs in other solvents (Fig. 2a), acetonitrile is simply evaporated and the desired solvent is added (chlorobenzene, dichlorobenzene, acetone, pyridine, tetrahydrofurane and so on). The proposed methodology of preparing supramolecular assemblies from inorganic-capped NCs and macrocycle molecules is very versatile. In addition to $\mathrm{PbS}$, we also successfully tested NCs of CdSe $(3 \mathrm{~nm})$, metals ( $\mathrm{Au}$ and $\mathrm{Pd}, 3-4 \mathrm{~nm}$ ) and core-shell $\mathrm{PbS} /$ $\mathrm{CdS}$ and $\mathrm{CdSe} / \mathrm{CdS}$ NCs. These NCs were combined with either $\mathrm{K}_{3} \mathrm{AsS}_{4}$ ligands or with $\mathrm{K}_{4} \mathrm{SnS}_{4}$ and $\mathrm{K}_{4} \mathrm{GeS}_{4}$. In addition to decyl-18C6, a variety of commercially available macrocycles were also tested: dicyclohexano-18-crown-6 (DCH-18C6, from Merck), dibenzo-18-crown-6 (DB-18C6, from Merck), $4^{\prime}, 4^{\prime \prime}\left(5^{\prime \prime}\right)$-di-tertbutyldicyclohexano-18-crown-6 (DTB-DCH-18C6, from Aldrich, see Fig. 1), 1,10-didecyl-1, 10-diaza-18-crown-6 (DD-diaza-18C6, from Aldrich), 4,7, 13,16,21,24-hexaoxa-1,10-diazabicyclo[8.8.8]hexacosane (cryptand C222, from ABCR, see Fig. 1) and 5,6-benzo4,7,13,16,21,24-hexaoxa-1,10-diazabicyclo[8.8.8] hexacos-5-ene (benzyl-C222, from Aldrich). It is worth noting that a variety of alkylated lipophilic derivatives of cryptands have been reported in the literature and may be of great interest as well, such as $\mathrm{C}_{10^{-}}$ and $\mathrm{C}_{14}$-grafted $\mathrm{C} 222$ cryptands ${ }^{48}$. All tested macrocycles allow the transfer of NCs into solvents of lower polarity $(\varepsilon=2-40)$ than the solvents in which pristine inorganic-capped NCs can initially be dispersed $(\varepsilon=40-191$, the highest for MFA and
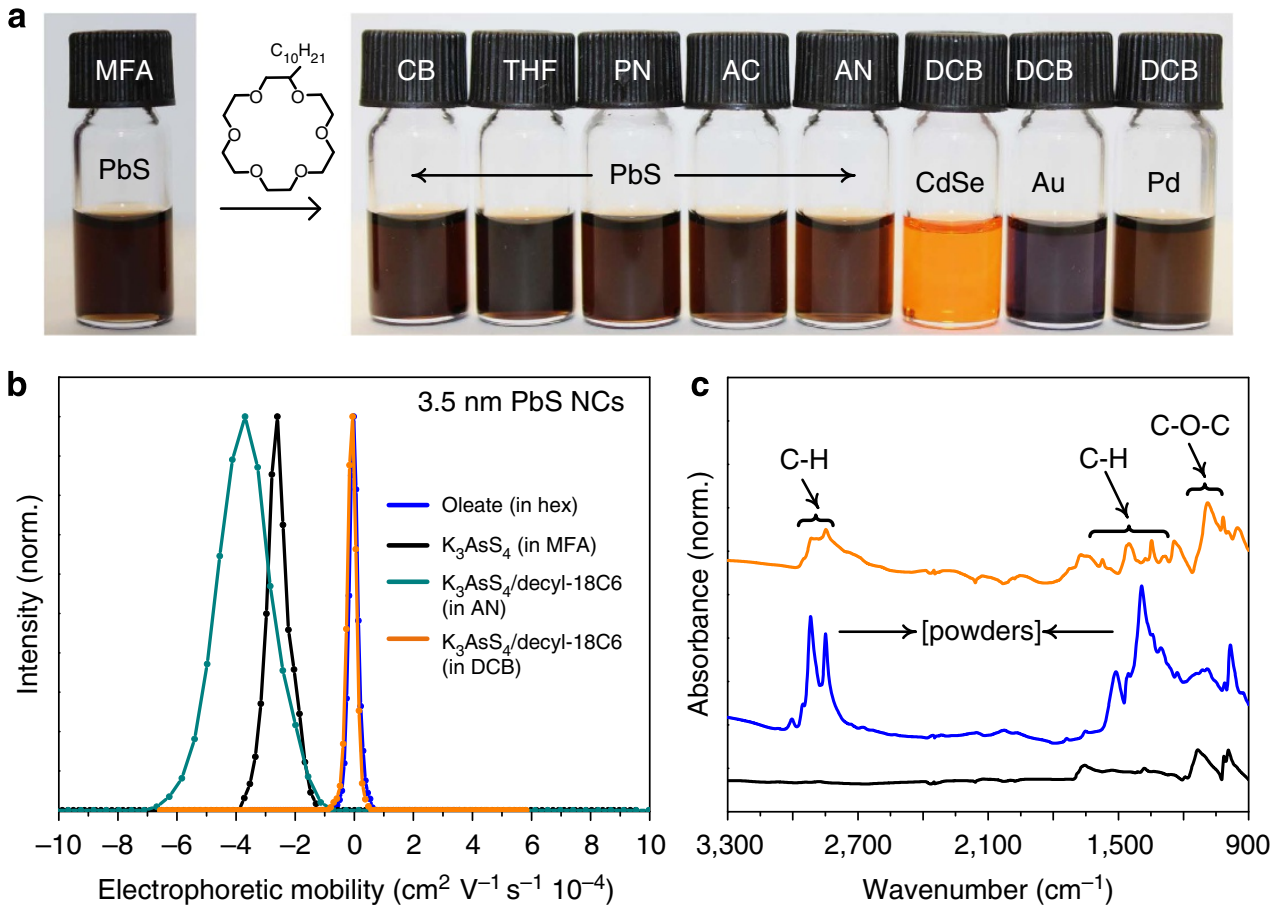

Figure 2 | Dispersibility tuning of inorganic-capped colloidal NCs using a host-guest supramolecular approach. (a) Stable colloidal solutions of various metallic and semiconductor $\mathrm{K}_{3} \mathrm{AsS}_{4}$-capped $\mathrm{NCs}$ in common polar and nonpolar solvents, obtained by solubilizing with decyl-18C6; (b) electrophoretic mobility measurements for oleate-, $\mathrm{K}_{3} \mathrm{AsS}_{4}-$, and $\mathrm{K}_{3} \mathrm{AsS}_{4}$ /decyl-18C6-capped PbS NCs, and (c) the corresponding FTIR spectra of powdered samples. Solvents: MFA, N-methylformamide; $C B$, chlorobenzene; THF, tetrahydrofurane; PN, pyridine; $A C$, acetone; $A N$, acetonitrile; $D C B$, dichlorobenzene. 
$N$-methylacetamide). As expected, access to rather nonpolar solvents such as CB and DCB is provided only by macrocycles containing aliphatic and aromatic side groups, with DTB-DCH$18 \mathrm{C} 6$, and decyl-18C6 being the most successful among the examples tested herein.

Electrophoretic mobility measurements (Fig. 2b) indicate that with the addition of decyl-18-crown- 6 onto the surface of the $\mathrm{NCs}$, followed by redispersion in a solvent of lower polarity $(\varepsilon<10$, for example, chlorobenzene, toluene and so on), the effective surface charge (measured as zeta-potential) is reduced due to ion pairing between the ligand anions and counter cations. In addition, a steric component is provided by the decyl chain. In a solvent of medium polarity, such as acetonitrile (acetonitrile, $\varepsilon=37.5$ ), no ion pairing occurs and instead a highly negative surface potential is observed; and the role of the macrocycle in this case is to aid the electrostatic dissociation of the cation from the NC surface. In fact, corresponding zeta-potentials of $\mathrm{PbS} / \mathrm{K}_{3} \mathrm{AsS}_{4} \mathrm{NCs}$ in MFA and $\mathrm{PbS} / \mathrm{K}_{3} \mathrm{AsS}_{4} /$ decyl-18C6 NCs in acetonitrile are similarly high: ca. $-50 \mathrm{~V}$ (Supplementary Fig. 1). These examples illustrate the tunable amphiphilicity provided by the macrocycle and its dependence upon the solvent. The true colloidal nature of the obtained solutions is confirmed by dynamic light scattering measurements, showing single-particle populations (Supplementary Fig. 2) and the absence of any macroscopic aggregates. The slightly larger hydrodynamic radius of $\mathrm{PbS} / \mathrm{K}_{3} \mathrm{AsS}_{4} /$ decyl-18C6 compared to initial and ligandexchanged colloids indicate the contribution from the corona of macrocycles. Fourier-transform infrared (FTIR) spectra of powdered samples verify the removal of the oleate ligand and, after ligand exchange, the attachment of the macrocycle forming $\mathrm{PbS} / \mathrm{K}_{3} \mathrm{AsS}_{4} /$ decyl-18C6 (Fig. 2c). Elemental analysis by the RBS (Supplementary Table 1) indicates that the $\mathrm{K}_{3} \mathrm{AsS}_{4}$ capping layer remains intact (that is, does not desorb from the NC surface) throughout the subsequent manipulations with macrocycles and solvents. A direct evidence for the coordination of macrocycle to the NC surface is provided by the ${ }^{1} \mathrm{H}$ nuclear magnetic resonance (NMR) spectroscopy (Supplementary Figs 3 and 4, and related discussion). Binding of the organic moieties to the NC surface results in significant broadening of solution NMR signals due to slower tumbling and, therefore, slower relaxation of molecule, smearing out the identity of the NMR peaks. Etching of the NCs by a dilute acid liberates the macrocycle and restores sharp features in the NMR signal.

As a result of addition of macrocycles, stable NC dispersions can be obtained in solvents of medium polarity (acetonitrile, acetone, pyridine and so on) which are traditionally inaccessible for purely charge- or purely sterically stabilized colloids. Previously, the solubilization of MCC-capped NCs in highly nonpolar solvents (toluene and chloroform) has been achieved via the exchange of alkali metal cations with bulky alkylammonium cations, such as didodecyldimethylammonium bromide $(\mathrm{DDAB})^{9}$; however, dispersibility in solvents of medium polarity was not achieved. The resulting DDA-inorganic ligand-NC assembly features a strongly bound DDA-ligand ionic pair that can be dissociated only by highly invasive chemical means such as solid-state cation exchange or thermal decomposition. The host-guest supramolecular approach presented here does not establish any direct, strong interaction between the macrocycle and inorganic ligand and is much easier to dissociate when needed.

Self-organization of 'crowned' nanocrystals. An important problem in NC self-assembly is balancing the entropic factors (symmetry and packing density) with specific interactions such as Coulomb interactions of surface charges, dipole-dipole interactions and van der Waals forces. Colloids of inorganiccapped NCs are stabilized electrostatically, for example, through the formation of an electric double layer. Because of strong longrange

electrostatic repulsion, such NCs rarely pack into long-range ordered structures, presumably because of a suppressed ability to carry out dynamic structural reorganization at short distances at a late stage of solvent evaporation. In contrast, short-range steric stabilization of organically passivated NCs allows the facile growth of various long-range ordered superlattices from nonpolar organic solvents upon simple drying ${ }^{49,50}$. Both entropy and weak short-range energetic interactions (dispersion forces and dipoledipole interactions) govern the assembly of colloidal $\mathrm{NCs}^{51-53}$, contributing to the change in Gibbs free energy of the system: $\mathrm{d} G=\mathrm{d} H-T \mathrm{~d} S$. The gain in free volume entropy upon ordering is greater than the decrease in configurational entropy, thus providing a net positive change to the system's entropy and driving assembly into close-packed structures. In full agreement with these arguments, a transmission electron microscopy (TEM) study of the NC films obtained from all tested solvents shows that indeed only the charge-neutral, sterically stabilized NCs in nonpolar solvents form long-range ordered superlattices upon solvent evaporation (Fig. 3). NCs smaller than $10 \mathrm{~nm}$ (PbS and Pd, Fig. 3a-c,f) tend to form a hexagonal close-packed lattice, while larger, cubic-shaped PbS NCs (Fig. 3d,e) form a body-centred cubic lattice.

Photoconductive properties of 'crowned' PbS NCs. In the past several years, $\mathrm{PbS}$ NCs have become exceptional materials for optoelectronic applications, showing promise for photovoltaics ${ }^{3,6}$ as well as for photon detection in the visible and infrared spectral regions ${ }^{2,54-57}$. In photoconductive devices, both an extremely high light responsivity (outperforming commercial devices) ${ }^{2}$ and a high speed of response to light ${ }^{56,58}$ have been reported. A general trend for most photoconductors is that higher sensitivities are accompanied by slower response (that is, a smaller bandwidth $B)$. This is consistent with the key role played by charge trapping states, controlled mainly by the surface chemistry, that are responsible for photoconductive gain effects in photoactive nanocrystalline materials ${ }^{59}$. The photoconductive gain $(G)$ is the ratio between de-trapping and transit times of localized and mobile carriers, respectively. Thus, long-lived traps lead to multiple passes of mobile carriers through the device, increasing $G^{1}$. De-trapping time is known to increase with the depth of the trap states, with respect to the bottom of the conduction band (for electrons) or top of the valence band (for holes), in both bulk materials ${ }^{60}$ and in $\mathrm{NCs}^{58}$. For PbS NCs, an empirical figure of merit $G \times B$ can be used. When $G$ and $B$ are measured under similar, ideally low light intensity, the $G \times B$ product for optimized $\mathrm{PbS} \mathrm{QD}$ photodetectors should be in the range of $10^{4}-10^{5} \mathrm{~Hz}$ (refs 57,58). Hence for the rational engineering of photodetector characteristics (sensitivity, detectivity and response time) control over the depth and density of trap states is critical ${ }^{57}$. Judicious choice of inorganic capping ligands enables control over the trapping/de-trapping dynamics. Here we show that also the organic corona of macrocycles can be used to tune $G$ and $B$, while preserving the expected $G \times B$ product. The observations described below can be attributed to the combined effect of the improved short-to-medium range order in the arrangement of the NCs within the film, and adjustments of the mean interparticle spacing.

The resulting conductive and photoconductive properties of NC-ligand-macrocycle films are governed by all three adjustable components: composition of a NC, selection of inorganic ligand and macrocycle. Films of oleate-capped $3 \mathrm{~nm}$ PbS NCs are highly 

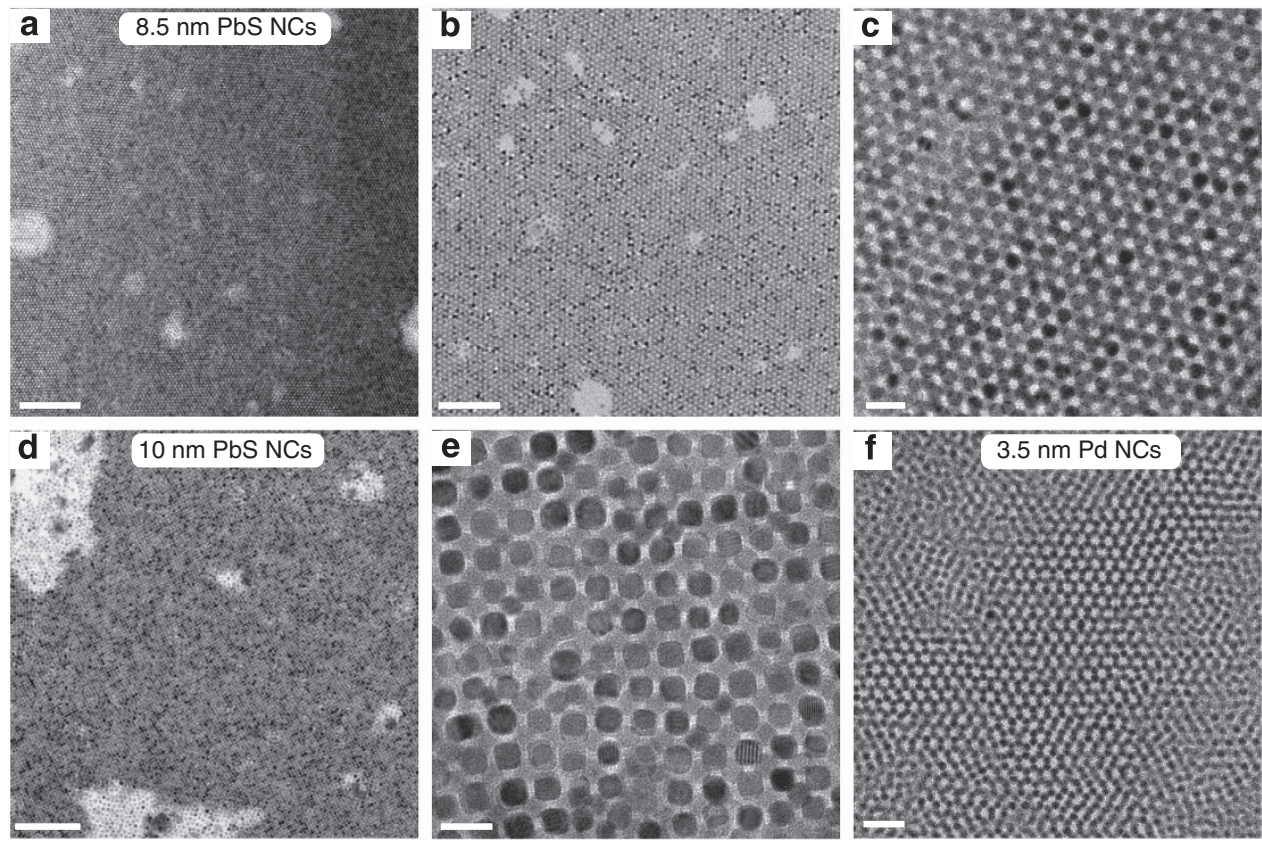

Figure 3 | Host-guest chemistry enables self-organization of inorganic-capped NCs into long-range ordered superlattices. TEM images of superlattices prepared from $\mathrm{K}_{3} \mathrm{AsS}_{4} /$ decyl-18-crown-6-capped (a-c) $8.5 \mathrm{~nm}$ PbS NCs, (d,e) $10 \mathrm{~nm}$ PbS NCs and (f) $3.5 \mathrm{~nm}$ Pd NCs. Scale bars are $200 \mathrm{~nm}$ (a), $100 \mathrm{~nm}$ (b), $20 \mathrm{~nm}(\mathbf{c}), 200 \mathrm{~nm}(\mathbf{d}), 20 \mathrm{~nm}(\mathbf{e}), 20 \mathrm{~nm}(\mathbf{f})$.

insulating, with conductivities typically below $10^{-11} \mathrm{Scm}^{-1}$. $\mathrm{K}_{3} \mathrm{AsS}_{4} /$ decyl-18C6 or $\mathrm{K}_{3} \mathrm{AsS}_{4} / \mathrm{C} 222$ capping renders $\mathrm{PbS} \mathrm{NC}$ films $10^{4}$-fold more conductive: 0.2 and $0.1 \mu \mathrm{S} \mathrm{cm}^{-1}$, respectively. Fully inorganic capping with only $\mathrm{K}_{3} \mathrm{AsS}_{4}$ leads to even higher conductivities of up to $25 \mu \mathrm{Scm}^{-1}$, confirming the prevailing role of the ligand capping for charge transport. In the case of equally sized metallic Pd NCs, a further increase in conductivity is observed with both $\mathrm{K}_{3} \mathrm{AsS}_{4} /$ decyl-18C6 capping and with $\mathrm{K}_{3} \mathrm{AsS}_{4}$ as the sole ligand $\left(20\right.$ and $\sim 100 \mathrm{Scm}^{-1}$, respectively). This demonstrates the significance of the NC core, presumably contributing mainly to the overall carrier density of the composite, with metals offering the highest number of mobile carriers. $\mathrm{AsS}_{4}{ }^{3-}$ is electronically insulating by itself, but owing to its small size, a thin capping layer of $\mathrm{K}_{3} \mathrm{AsS}_{4}$ mediates tunnelling and charge hopping within the NC film. While these general observations shed important insight on the conductive properties of NC-ligand-macrocycle films, charge transport in NC solids is a matter of high complexity and for more detail the reader is referred elsewhere ${ }^{1,6,7,61}$.

For photoconductivity experiments, $\mathrm{PbS} \mathrm{NCs} / \mathrm{K}_{3} \mathrm{AsS}_{4} /$ decyl18C6 colloids were drop casted over interdigitated electrodes with $10 \mu \mathrm{m}$ gaps and a total channel length of $43 \mathrm{~mm}$ (Fig. 4a). Upon illumination, $\mathrm{PbS}$ NC films exhibit high photocurrent, which is 100-1,000 times higher than the current in darkness (Fig. 4b). The responsivity spectrum (Fig. 4c) closely matches the nearinfrared absorption spectrum, with a pronounced excitonic peak. The responsivity exhibits a highly linear dependence on the incident light intensity (Fig. 4d). The responsivity value of $\sim 40 \mathrm{~A} \mathrm{~W}^{-1}$ can be estimated at the excitonic peak of $1,200 \mathrm{~nm}$, yielding $G$ of at least 40 . With the noise equivalent power of $\sim 2 \times 10^{-13} \mathrm{~W} \mathrm{~Hz}^{-1 / 2}$, a high detectivity of $3.3 \times 10^{11}$ Jones is estimated at a wavelength of $1,200 \mathrm{~nm}$. A value of $G \geq 1$ indicates the localization of one carrier type in trap states. Sulfidoarsenate and other chalcogenide ligands were previously reported to act as hole traps ${ }^{57}$. For instance, highly electronically passivated oleatecapped $\mathrm{PbS}$ NCs initially exhibit high-photoluminescence quantum yields of up to $40 \%$ and long radiative lifetimes on the order of hundreds of nanoseconds (Fig. 4e). Upon exchange with $\mathrm{K}_{3} \mathrm{AsS}_{4}$, photoluminescence quantum yields decrease by 1-2 orders of magnitude and average photoluminescence lifetimes are as short as one to several nanoseconds, in both solutions and films, with and without macrocycles attached (Supplementary Fig. 5). Hence, we conclude that trapping of holes does play an important role in this study as well and is a primary charge carrier trapping mechanism.

The de-trapping time is revealed by the frequency dependence of the photoresponse (Fig. 4f) and, as discussed above, provides a trade-off between the speed and sensitivity of the detector. $\mathrm{A} 3 \mathrm{~dB}$ cut-off is located at $\sim 3 \mathrm{kHz}$. For comparison, we have recently reported highly sensitive all-inorganic $\mathrm{PbS} / \mathrm{As}_{2} \mathrm{~S}_{3}$ photodetectors with higher sensitivities $\left(200 \mathrm{~A} \mathrm{~W}^{-1}\right)$ and detectivities $\left(2 \times 10^{13}\right.$ Jones) at the same wavelengths, but with a sacrifice of the response times; correspondingly, these exhibited a slow frequency response with a $3 \mathrm{~dB}$ value of just $10 \mathrm{~Hz} . G \times B$ products of both detectors are $1-2 \times 10^{5} \mathrm{~Hz}$. The much faster photoresponse of $\mathrm{PbS} / \mathrm{K}_{3} \mathrm{AsS}_{4} /$ decyl-18C6 films can be explained by the fact that discrete $\mathrm{AsS}_{4}{ }^{3-}$ has a wider gap between the highest occupied molecular orbital and lowest unoccupied molecular orbital (HOMO-LUMO gap) and does not introduce as many nor such deep trap states as the much narrower gap amorphous $\mathrm{As}_{2} \mathrm{~S}_{3}$ semiconducting capping layer generated by decomposing $\left(\mathrm{NH}_{4}\right)_{3} \mathrm{AsS}_{3}$ ligands in our previous work ${ }^{57}$.

\section{Discussion}

This study shows that weak (non-covalent and non-ionic) structure-specific interactions of high selectivity can lead to very useful adjustments of the chemical and physical properties of colloidal inorganic NCs and their solids. NC-ligand-crown and NC-ligand-cryptand assemblies exhibit broadly tunable dispersibility in various solvents, behaving as amphiphilic moieties due to both steric and electrostatic contributions to the interparticle interactions. This approach also assists in self-organization of NCs by switching the repulsive forces responsible for colloidal stabilization of inorganically capped NCs from long-range electrostatic to short-range steric. Besides 
a

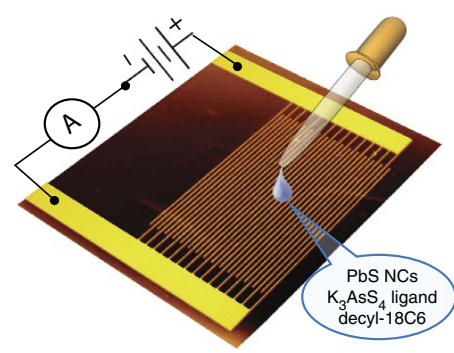

c

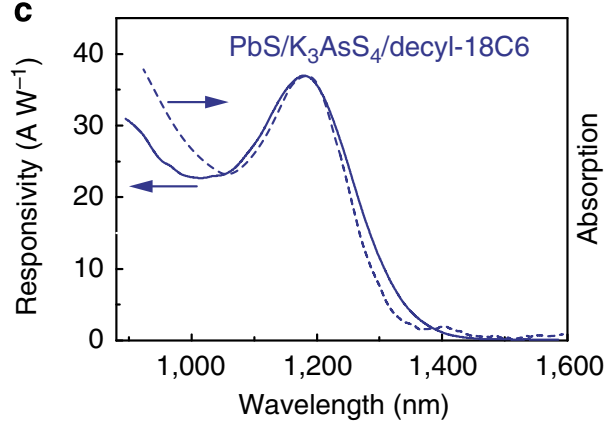

e

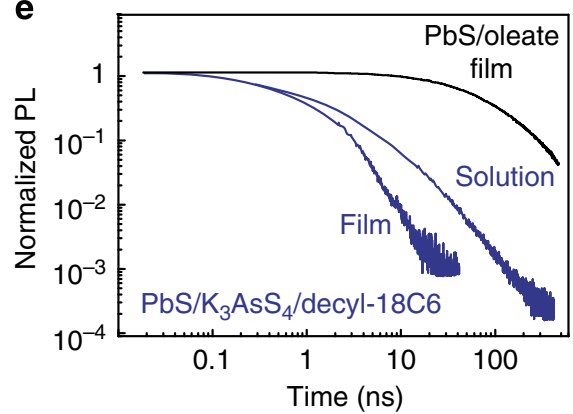

b

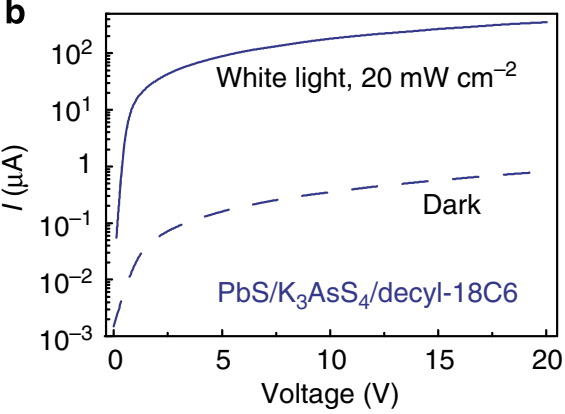

d

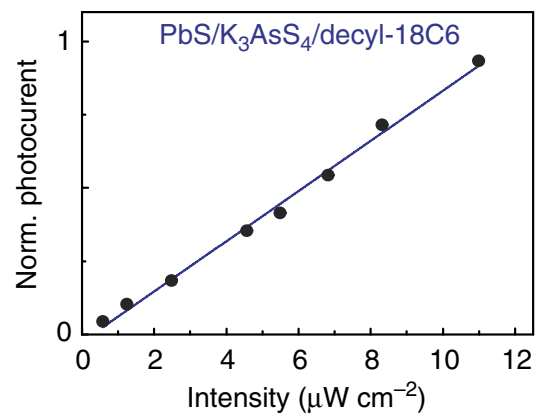

f

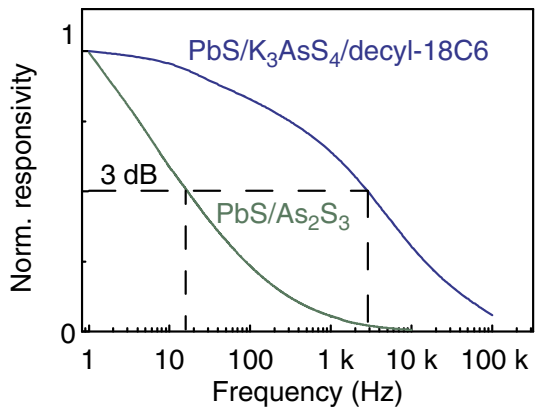

Figure 4 | Photoconductive properties of 'crowned' PbS NCs. (a) Schematic of a solution processed photodetector; the pseudo-three-dimensional image of a glass substrate with interdigitated gold electrodes was rendered with Gwyddion software from a microphotograph of a real device; (b) current-voltage characteristics for $\sim 3 \mathrm{~nm} \mathrm{PbS} \mathrm{NCs} \mathrm{capped} \mathrm{with} \mathrm{K}_{3} \mathrm{AsS}_{4} /$ decyl-18C6 in darkness (dashed line) and under white-light illumination of $20 \mathrm{~mW} \mathrm{~cm}^{-2}$ (solid line); (c) responsivity spectrum of the same film; (d) intensity dependence of the photocurrent during illumination at the excitonic peak $(\lambda \approx 1,200 \mathrm{~nm})$; (e) time-resolved photoluminescence decays from films and solutions before and after ligand-exchange; (f) frequency dependence of the photocurrent for $\mathrm{PbS} N C s$ capped with $\mathrm{K}_{3} \mathrm{AsS}_{4} / \mathrm{DC}$ (blue) and PbS NCs with an amorphous $\mathrm{As}_{2} \mathrm{~S}_{3}$ capping layer (green). Norm., normalized.

acting as surface-active reagents, macrocycles also have a profound effect on the electronic properties of NC solids. For instance, high responsivities of up to $40 \mathrm{~A} \mathrm{~W}^{-1}$ and a high speed of operation (bandwidth of $3 \mathrm{kHz}$ ) can be demonstrated. While here we only utilized commercially available crown ethers, cryptands and their derivatives, a variety of other macrocycles may also potentially be used for such purposes. Examples of the latter include calixarenes, acyclic molecules such as oligo-ethylene-glycols, and many natural molecules (for example, valinomycin) that exhibit a known alkali metal-specific binding.

\section{Methods}

Synthesis of NCs. PbS NCs (3-11 nm) capped with oleic acid were synthesized from lead oleate and bis(trimethylsilyl)sulfide as reported elsewhere ${ }^{62}$. The size of the PbS NCs was controlled by varying the molar ratio of oleic acid to octadecene. Wurtzite-phase CdSe NCs $(3 \mathrm{~nm})$ were synthesized according to the procedure reported by the Bawendi group ${ }^{63}$. OAm-capped Au NCs $(3.8 \mathrm{~nm})^{64}$ and Pd NCs $(4 \mathrm{~nm})^{65}$ were also synthesized as reported elsewhere.

Ligand exchange with $\mathbf{K}_{\mathbf{3}} \mathbf{A s S}_{\mathbf{4}} \cdot \mathrm{K}_{3} \mathrm{AsS}_{4}$ was prepared by mixing stoichiometric quantities of $\mathrm{As}_{2} \mathrm{~S}_{5}$ and $\mathrm{K}_{2} \mathrm{~S}$ in water for $10 \mathrm{~h}$ and isolated by adding acetonitrile, followed by drying. Freshly prepared $\mathrm{K}_{3} \mathrm{AsS}_{4}(5 \mathrm{mg})$ was dissolved in $1 \mathrm{ml}$ of MFA and mixed with $5 \mathrm{mg}$ of PbS NCs dispersed in $1 \mathrm{ml}$ of hexane. The two-phase system was stirred vigorously for $1 \mathrm{~h}$. The NCs in MFA were rinsed three times with

5-6 $\mathrm{ml}$ of hexane and filtered through a $0.2 \mu \mathrm{m}$ PTFE filter.

Host-guest chemistry with macrocycles. NCs in MFA $\left(100 \mu \mathrm{l}, 5 \mathrm{mg} \mathrm{ml}^{-1}\right)$ were precipitated by the addition of $0.4-1 \mathrm{ml}$ of toluene or toluene/acetonitrile mixture, followed by centrifugation. Then, $40-100 \mu \mathrm{l}$ of the solution of macrocyclic polyethers in acetonitrile $(0.05-0.1 \mathrm{M})$ were added to redisperse the NCs. Acetonitrile was removed by drying under vacuum. The NCs were then redispersed in DCB. To remove excess macrocycles, the NCs were precipitated with $0.1-0.5 \mathrm{ml}$ of ethanol or an ethanol/hexane mixture. Thus obtained NCs were fully dispersible in nonpolar solvents such as $\mathrm{CB}, \mathrm{DCB}, \mathrm{THF}, \mathrm{PN}$ and so on.

Preparation of NC superlattices. A solution of NC-macrocycle assemblies in $\mathrm{CB}$ or DCB $(20-30 \mu \mathrm{l})$ with a NC concentration of $\sim 10^{14}-10^{15} \mathrm{NCs}$ per ml was placed onto a carbon-coated TEM grid and dried at $50-55^{\circ} \mathrm{C}$ in the closed chamber under nitrogen for several hours.

Characterization. TEM images were recorded using a JEOL JEM-2200FS microscope operated at $200 \mathrm{kV}$. Electrophoretic mobility and dynamic light scattering measurements were performed using a Zetasizer Nano-ZS instrument (Malvern Instruments, Inc.). Colloidal solutions were tested using a dip-cell setup with Pd electrodes. FTIR spectra were measured using a Thermo Scientific Nicolet iS5 FTIR spectrometer. RBS was performed at the ETH Laboratory of Ion Beam 
Physics. Measurements were conducted using a $2 \mathrm{MeV}^{4} \mathrm{He}$ beam and a silicon PIN diode detector under an angle of $168^{\circ}$. The collected RBS data were analysed using simulations by the RUMP code ${ }^{66}$. NMR spectra were acquired on a Bruker $11.7 \mathrm{~T}$ spectrometer equipped with $\mathrm{PABBO}$ gradient field three channel liquid-state probe head for $5 \mathrm{~mm}$ NMR tubes. Experiments were performed at room temperature in non-spinning mode while locking the deuterium signal of the $\mathrm{CD}_{3} \mathrm{CN}$. The $90^{\circ}$ pulse and the recovery delay for ${ }^{1} \mathrm{H}$ was $11.40 \mu$ s and $5 \mathrm{~s}$, respectively $9.50 \mu$ s and $2 \mathrm{~s}$ for ${ }^{13} \mathrm{C}$. One-dimensional spectra were acquired with a single pulse sequence acquiring 1 scan for ${ }^{1} \mathrm{H}$ and 1,024 scans for ${ }^{13} \mathrm{C}$. Chemical shifts were referenced to $\mathrm{Si}\left(\mathrm{CH}_{3}\right)_{4}$ as an external standard.

Photoconductivity measurements were performed with illumination from a tungsten lamp dispersed by an Acton SP2150 (Roper Scientific) spectrograph/ monochromator. For measurements of the responsivity spectra, the light was modulated by a mechanical chopper at a frequency of $33 \mathrm{~Hz}$. The sample was biased using a Keithley 236 SMU by $10-20$ V, limited to obtain stable dark currents in the range of $1-2 \mu \mathrm{A}$. The signal, amplitude and phase were measured across a series resistant by a Stanford Research 830 lock-in amplifier. The light intensity was controlled by a calibrated power detector (UM9B-BL, Gentec-EO). Currentvoltage $(I-V)$ characteristics were also measured by the Keithley 236 SMU. Frequency dependencies were obtained with a load resistance of $1,000 \Omega$, under pulsed and focused illumination from an LED emitting at a wavelength of $1,200 \mathrm{~nm}$.

Time-resolved photoluminescence traces in the infrared region were obtained by a Time-Correlated Single Photon Counting (TCSPC) system based on an InGaAs TE-cooled single-photon avalanche photodiode (ID Quantique) with 200 ps time resolution, adjusted for $10 \%$ quantum efficiency, using a SPC-130-EM Counting Module and BDL-488-SMN laser (Becker \& Hickl) with a pulse duration of $50 \mathrm{ps}$ and wavelength of $488 \mathrm{~nm}$, and with a CW power equivalent of $\sim 0.5 \mathrm{~mW}$, externally triggered at a $1 \mathrm{MHz}$ repetition rate. photoluminescence emission from the samples passed through a long-pass optical filter with an edge at $900 \mathrm{~nm}$.

\section{References}

1. Talapin, D. V., Lee, J. S., Kovalenko, M. V. \& Shevchenko, E. V. Prospects of colloidal nanocrystals for electronic and optoelectronic applications. Chem. Rev. 110, 389-458 (2010).

2. Konstantatos, G. et al. Ultrasensitive solution-cast quantum dot photodetectors. Nature 442, 180-183 (2006).

3. Chuang, C.-H. M., Brown, P. R., Bulović, V. \& Bawendi, M. G. Improved performance and stability in quantum dot solar cells through band alignment engineering. Nat. Mater. 13, 796-801 (2014).

4. Liu, Y. et al. PbSe quantum dot field-effect transistors with air-stable electron mobilities above $7 \mathrm{~cm}^{2} \mathrm{~V}^{-1} \mathrm{~s}^{-1}$. Nano Lett. 13, 1578-1587 (2013).

5. Kovalenko, M. V. et al. Prospects of nanoscience with nanocrystals. ACS Nano 9, 1012-1057 (2015).

6. Zhitomirsky, D. et al. Engineering colloidal quantum dot solids within and beyond the mobility-invariant regime. Nat. Commun. 5, 3803 (2014).

7. Guyot-Sionnest, P. Electrical transport in colloidal quantum dot films. J. Phys. Chem. Lett. 3, 1169-1175 (2012).

8. Kovalenko, M. V., Scheele, M. \& Talapin, D. V. Colloidal nanocrystals with molecular metal chalcogenide surface ligands. Science 324, 1417-1420 (2009).

9. Kovalenko, M. V., Bodnarchuk, M. I. \& Talapin, D. V. Nanocrystal superlattices with thermally degradable hybrid inorganic - organic capping ligands. J. Am. Chem. Soc. 132, 15124-15126 (2010).

10. Lee, J. E. et al. Facile and economical synthesis of hierarchical carbon-coated magnetite nanocomposite particles and their applications in lithium ion battery anodes. Energy Environ. Sci. 5, 9528-9533 (2012).

11. Jiang, C., Lee, J.-S. \& Talapin, D. V. Soluble precursors for CuInSe 2 , CuIn ${ }_{1-x}$ $\mathrm{Ga}_{x} \mathrm{Se}_{2}$, and $\mathrm{Cu}_{2} \mathrm{ZnSn}(\mathrm{S}, \mathrm{Se})_{4}$ based on colloidal nanocrystals and molecular metal chalcogenide surface ligands. J. Am. Chem. Soc. 134, 5010-5013 (2012).

12. Liu, W., Lee, J. S. \& Talapin, D. V. III-V nanocrystals capped with molecular metal chalcogenide ligands: high electron mobility and ambipolar photoresponse. J. Am. Chem. Soc. 135, 1349-1357 (2013).

13. Kovalenko, M. V., Schaller, R. D., Jarzab, D., Loi, M. A. \& Talapin, D. V. Inorganically functionalized PbS-CdS colloidal nanocrystals: integration into amorphous chalcogenide glass and luminescent properties. J. Am. Chem. Soc 134, 2457-2460 (2012).

14. Tsokkou, D. et al. Photophysics of PbS quantum dot films capped with arsenic sulfide ligands. Adv. Energy Mater. 4, 1301547 (2014).

15. Ocier, C. R., Whitham, K., Hanrath, T. \& Robinson, R. D. Chalcogenidometallate clusters as surface ligands for PbSe nanocrystal fieldeffect transistors. J. Phys. Chem. C 118, 3377-3385 (2014).

16. Jang, J., Liu, W., Son, J. S. \& Talapin, D. V. Temperature-dependent hall and field-effect mobility in strongly coupled all-inorganic nanocrystal arrays. Nano Lett. 14, 653-662 (2014).

17. Nag, A. et al. Effect of metal ions on photoluminescence, charge transport, magnetic and catalytic properties of all-inorganic colloidal nanocrystals and nanocrystal solids. J. Am. Chem. Soc. 134, 13604-13615 (2012).

18. Kovalenko, M. V., Bodnarchuk, M. I., Zaumseil, J., Lee, J.-S. \& Talapin, D. V. Expanding the chemical versatility of colloidal nanocrystals capped with molecular metal chalcogenide ligands. J. Am. Chem. Soc. 132, 10085-10092 (2010).

19. Tangirala, R., Baker, J. L., Alivisatos, A. P. \& Milliron, D. J. Modular inorganic nanocomposites by conversion of nanocrystal superlattices. Angew. Chem. Int. Ed. 49, 2878-2882 (2010).

20. Kovalenko, M. V. et al. Semiconductor nanocrystals functionalized with antimony telluride zintl ions for nanostructured thermoelectrics. J. Am. Chem Soc. 132, 6686-6695 (2010).

21. Dirin, D. N. et al. Lead halide perovskites and other metal halide complexes as inorganic capping ligands for colloidal nanocrystals. J. Am. Chem. Soc. 136, 6550-6553 (2014).

22. Chung, D. S. et al. Low voltage, hysteresis free, and high mobility transistors from all-inorganic colloidal nanocrystals. Nano Lett. 12, 1813-1820 (2012).

23. Rowland, C. E. et al. Thermal stability of colloidal InP nanocrystals: small inorganic ligands boost high-temperature photoluminescence. ACS Nano $\mathbf{8}$, 977-985 (2013).

24. Zhang, H. et al. Surfactant ligand removal and rational fabrication of inorganically connected quantum dots. Nano Lett. 11, 5356-5361 (2011).

25. Kravchyk, K. et al. Monodisperse and inorganically capped $\mathrm{Sn}$ and $\mathrm{Sn} / \mathrm{SnO}_{2}$ nanocrystals for high-performance Li-ion battery anodes. J. Am. Chem. Soc. 135, 4199-4202 (2013).

26. Nag, A. et al. Metal-free inorganic ligands for colloidal nanocrystals: $\mathrm{S}^{2-}, \mathrm{HS}^{-}$, $\mathrm{Se}^{2-}, \mathrm{HSe}^{-}, \mathrm{Te}^{2-}, \mathrm{HTe}^{-}, \mathrm{TeS}_{3}^{2-}, \mathrm{OH}^{-}$, and $\mathrm{NH}^{2-}$ as Surface Ligands. J. Am. Chem. Soc. 133, 10612-10620 (2011).

27. Doris, S. E. et al. Mechanistic insight into the formation of cationic naked nanocrystals generated under equilibrium control. J. Am. Chem. Soc. 136, 15702-15710 (2014).

28. Rosen, E. L. et al. Exceptionally Mild reactive stripping of native ligands from nanocrystal surfaces by using Meerwein's salt. Angew. Chem. Int. Ed. 51, 684-689 (2012).

29. Choi, J.-H. et al. Bandlike transport in strongly coupled and doped quantum dot solids: a route to high-performance thin-film electronics. Nano Lett. 12, 2631-2638 (2012).

30. Kim, D. K., Lai, Y., Diroll, B. T., Murray, C. B. \& Kagan, C. R. Flexible and lowvoltage integrated circuits constructed from high-performance nanocrysal transistors. Nat. Commun. 3, 1216 (2012).

31. Oh, S. J. et al. Stoichiometric control of lead chalcogenide nanocrystal solids to enhance their electronic and optoelectronic device performance. ACS Nano 7, 2413-2421 (2013).

32. Oh, S. J. et al. Designing high-performance $\mathrm{PbS}$ and $\mathrm{PbSe}$ nanocrystal electronic devices through stepwise, post-synthesis, colloidal atomic layer deposition. Nano Lett. 14, 1559-1566 (2014).

33. Lee, J. S., Kovalenko, M. V., Huang, J., Chung, D. S. \& Talapin, D. V. Band-like transport, high electron mobility and high photoconductivity in all-inorganic nanocrystal arrays. Nat. Nanotechnol. 6, 348-352 (2011).

34. Munro, A. M., Jen-La Plante, I., Ng, M. S. \& Ginger, D. S. Quantitative study of the effects of surface ligand concentration on CdSe nanocrystal photoluminescence. J. Phys. Chem. C 111, 6220-6227 (2007).

35. Liao, J. H., Varotsis, C. \& Kanatzidis, M. G. Syntheses, structures, and properties of six novel alkali-metal tin sulfides - $\mathrm{K}_{2} \mathrm{Sn}_{2} \mathrm{~S}_{8}$, alpha- $\mathrm{Rb}_{2} \mathrm{Sn}_{2} \mathrm{~S}_{8}$, beta- $\mathrm{Rb}_{2} \mathrm{Sn}_{2} \mathrm{~S}_{8}, \mathrm{~K}_{2} \mathrm{Sn}_{2} \mathrm{~S}_{5}, \mathrm{Cs}_{2} \mathrm{~S}_{2} \mathrm{~S}_{6}$, and $\mathrm{Cs}_{2} \mathrm{SnS}_{14}$. Inorg. Chem. 32, 2453-2462 (1993).

36. Ning, Z. et al. Air-stable n-type colloidal quantum dot solids. Nat. Mater. 13, 822-828 (2014).

37. Dolzhnikov, D. S. et al. Composition-matched molecular 'solders' for semiconductors. Science 347, 425-428 (2015).

38. Wang, R. Y., Tangirala, R., Raoux, S., Jordan-Sweet, J. L. \& Milliron, D. J. Ionic and electronic transport in $\mathrm{Ag}_{2} \mathrm{~S}$ nanocrystal-GeS $\mathrm{S}_{2}$ matrix composites with size-controlled $\mathrm{Ag}_{2} \mathrm{~S}$ nanocrystals. Adv. Mater. 24, 99-103 (2012).

39. Ko, D.-K., Kang, Y. \& Murray, C. B. Enhanced thermopower via carrier energy filtering in solution processable $\mathrm{Pt}-\mathrm{Sb}_{2} \mathrm{Te}_{3}$ nanocomposites. Nano Lett. 11, 2841-2844 (2011).

40. Gargas, D. J. et al. Engineering bright sub-10-nm upconverting nanocrystals for single-molecule imaging. Nat. Nanotechnol. 9, 300-305 (2014).

41. Llordes, A. et al. Polyoxometalates and colloidal nanocrystals as building blocks for metal oxide nanocomposite films. J. Mater. Chem. 21, 11631-11638 (2011).

42. Pedersen, C. J. Cyclic polyethers and their complexes with metal salts. J. Am. Chem. Soc. 89, 7017-7036 (1967).

43. Dietrich, B., Lehn, J. M. \& Sauvage, J. P. Diaza-polyoxa-macrocycles et macrobicycles. Tetrahedron Lett. 10, 2885-2888 (1969).

44. Liotta, C. L. \& Berkner, J. in Encyclopedia of Reagents for Organic Synthesis (John Wiley \& Sons, Ltd, 2001).

45. Guo, J. et al. Crown ethers in graphene. Nat. Commun. 5, 5389 (2014).

46. Lehn, J.-M. in Supramolecular Chemistry: Concepts and Perspectives (WileyVCH Verlag GmbH \& Co. KGaA, 2006).

47. Zherebetskyy, D. et al. Hydroxylation of the surface of PbS nanocrystals passivated with oleic acid. Science 344, 1380-1384 (2014). 
48. Lauricella, T. L. et al. Lipophilic derivatives of [2.2. 1]-and [2.2. 2] cryptands: thermodynamics of micellization of their alkali and alkaline-earth cryptates. J. Org. Chem. 56, 1524-1529 (1991).

49. Shevchenko, E. V., Talapin, D. V., Kotov, N. A., O’Brien, S. \& Murray, C. B. Structural diversity in binary nanoparticle superlattices. Nature 439, 55-59 (2006).

50. Dong, A., Chen, J., Vora, P. M., Kikkawa, J. M. \& Murray, C. B. Binary nanocrystal superlattice membranes self-assembled at the liquid-air interface. Nature 466, 474-477 (2010).

51. Bodnarchuk, M. I., Kovalenko, M. V., Heiss, W. \& Talapin, D. V. Energetic and entropic contributions to self-assembly of binary nanocrystal superlattices: temperature as the structuredirecting factor. J. Am. Chem. Soc. 132, 11967-11977 (2010)

52. Chen, Z. \& O'Brien, S. Structure direction of II-VI semiconductor quantum dot binary nanoparticle superlattices by tuning radius ratio. ACS Nano 2, 1219-1229 (2008)

53. Evers, W. H. et al. Entropy-driven formation of binary semiconductornanocrystal superlattices. Nano Lett. 10, 4235-4241 (2010).

54. Konstantatos, G., Clifford, J., Levina, L. \& Sargent, E. H. Sensitive solutionprocessed visible-wavelength photodetectors. Nat. Photon 1, 531-534 (2007).

55. Lhuillier, E., Keuleyan, S., Liu, H. \& Guyot-Sionnest, P. Mid-IR colloidal nanocrystals. Chem. Mater. 25, 1272-1282 (2013).

56. Clifford, J. P. et al. Fast, sensitive and spectrally tuneable colloidal quantum-dot photodetectors. Nat. Nanotechnol. 4, 40-44 (2009).

57. Yakunin, S. et al. High infrared photoconductivity in films of arsenic-sulfideencapsulated lead-sulfide nanocrystals. ACS Nano 8, 12883-12894 (2014).

58. Tang, J. et al. Colloidal-quantum-dot photovoltaics using atomic-ligand passivation. Nat. Mater. 10, 765-771 (2011).

59. Konstantatos, G., Levina, L., Fischer, A. \& Sargent, E. H. Engineering the temporal response of photoconductive photodetectors via selective introduction of surface trap states. Nano Lett. 8, 1446-1450 (2008).

60. Bube, R. H. Photoconductivity of Solids 273-302 (John Wiley \& Sons, 1978).

61. Yang, J. \& Wise, F. W. Effects of disorder on electronic properties of nanocrystal assemblies. J. Phys. Chem. C 119, 3338-3347 (2015).

62. Hines, M. A. \& Scholes, G. D. Colloidal PbS nanocrystals with size-tunable near-infrared emission: observation of post-synthesis self-narrowing of the particle size distribution. Adv. Mater. 15, 1844-1849 (2003).

63. Chen, O. et al. Compact high-quality CdSe-CdS core-shell nanocrystals with narrow emission linewidths and suppressed blinking. Nat. Mater. 12, 445-451 (2013).

64. Peng, S. et al. A facile synthesis of monodisperse Au nanoparticles and their catalysis of CO oxidation. Nano Res. 1, 229-234 (2008).
65. Kim, S. W. et al. Synthesis of monodisperse palladium nanoparticles. Nano Lett. 3, 1289-1291 (2003).

66. Doolittle, L. R. A semiautomatic algorithm for Rutherford backscattering analysis. Nucl. Instr. Meth. Phys. Res. 15, 227-231 (1986).

\section{Acknowledgements}

This work was financially supported by the European Union through FP7 (ERC Starting Grant NANOSOLID, GA No. 306733). M.B. acknowledges financial support from the Swiss National Science Foundation (Marie-Heim Vögtlin fellowship 139784 and Ambizione Energy fellowship PZENP2_154287). M.K. and M.B. are grateful to Prof. Dmitri V. Talapin for supporting early trials to solubilize nanocrystal-macrocycle adducts in various solvents (2010, University of Chicago). All authors thank Dr Dmitry Dirin for providing CdSe NCs, Dr M. Döbeli for RBS measurements, and Dr Nicholas Stadie for reading the manuscript. M.B. and M.K. acknowledge support of the Empa Electron Microscopy Center.

\section{Author contributions}

M.I.B. and M.V.K. conceived the work; M.I.B. synthesized and characterized all materials; S.Y. studied photoconductive properties; L.P. performed NMR measurements. All authors discussed the results and co-wrote the manuscript.

\section{Additional information}

Supplementary Information accompanies this paper at http://www.nature.com/ naturecommunications

Competing financial interests: The authors declare no competing financial interests.

Reprints and permission information is available online at http://npg.nature.com/ reprintsandpermissions/

How to cite this article: Bodnarchuk, M. I. et al. Host-guest chemistry for tuning colloidal solubility, self-organization and photoconductivity of inorganic-capped nanocrystals. Nat. Commun.6:10142 doi: 10.1038/ncomms10142 (2015).

This work is licensed under a Creative Commons Attribution 4.0 International License. The images or other third party material in this article are included in the article's Creative Commons license, unless indicated otherwise in the credit line; if the material is not included under the Creative Commons license, users will need to obtain permission from the license holder to reproduce the material. To view a copy of this license, visit http://creativecommons.org/licenses/by/4.0/ 